\title{
Surgical and nonsurgical approaches to small-size nonsmall cell lung cancer
}

\author{
Dirk De Ruysscher ${ }^{1,3}$, Kazuo Nakagawa ${ }^{2,3}$, Hisao Asamura² \\ Number 3 in the series "Challenges and Controversies in Thoracic Oncology" \\ Edited by J-P. Sculier, B. Besse and P. Van Schil

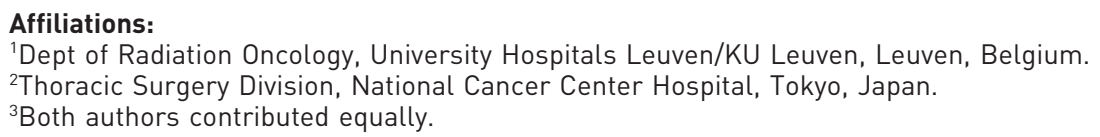

\section{Correspondence:}

Dirk De Ruysscher, Dept of Radiation Oncology, University Hospitals Leuven/KU Leuven, Herestraat 49, 3000 Leuven, Belgium.

E-mail: dirk.deruysscherduzleuven.be

ABSTRACT Lobectomy and systematic nodal dissection are still the standard for small-size $(<3 \mathrm{~cm})$ nonsmall cell lung cancer. There is growing interest in more parenchyma-sparing surgery, so-called sublobar resections (wedge resection or segmentectomy). Indeed, nonrandomised trials suggest that a segmentectomy may result in local control rates that are similar to lobectomy. Nonsurgical approaches, such as stereotactic ablative radiotherapy, consistently result in local control rates of $\sim 90 \%$ and survival rates that are comparable to lobectomy. Therefore, we are moving towards an era in which several therapeutic possibilities are available, that are probably equivalent from an oncological point of view. Further trials are needed to define the optimal therapy for individual patients. comparable to lobectomy http://ow.ly/x0Noc

\footnotetext{
Previous articles in this series: No. 1: Powell HA, Baldwin DR. Multidisciplinary team management in thoracic oncology: more than just a concept? Eur Respir J 2014; 43: 1776-1786; No. 2: Shlomi D, Ben-Avi R, Balmor GR, et al. Screening for lung cancer: time for large-scale screening by chest computed tomography. Eur Respir J 2014; 44: 217-238.

Received: Jan 302014 | Accepted after revision: May 082014 | First published online: June 122014

Conflict of interest: Disclosures can be found alongside the online version of this article at erj.ersjournals.com Copyright @ERS 2014
} 


\section{Introduction}

Nonsmall cell lung cancer (NSCLC) with a size of $3 \mathrm{~cm}$, at maximum, is traditionally treated with a lobectomy and mediastinal lymph node dissection. However, in recent years, there has been growing interest to consider less extensive and hence more lung-sparing surgery as well as nonsurgical approaches, such as stereotactic radiotherapy (SBRT) or stereotactic ablative radiotherapy (SABR) and radiofrequency ablation for these tumours.

In this review, we will give an overview of current data on which techniques may be used outside clinical trials. Indeed, in the absence of phase III randomised data and because neither sublobar resections nor SABR are clearly superior to another for the whole patient population; we have attempted to give a balanced view. Moreover, patient characteristics, tumour characteristics and local expertise should be taken into account for each individual patient.

\section{Surgical approaches}

Surgery remains the cornerstone of treatment for early stage NSCLC, and lobectomy, including systematic lymph node dissection, is considered the standard of care for patients with early stage NSCLC $[1,2]$. To date, sublobar resection is performed most often as an alternative to lobectomy in patients with peripheral tumours with limited pulmonary reserve or other comorbidities [3-6]. The increased ability to identify smaller NSCLC by enhanced computed tomography (CT) screening has led many surgeons to question the appropriateness of lobectomy in all patients with small-sized NSCLC [7]. Furthermore, the growing recognition that patients with smaller tumours are a favourable subset of patients with stage I NSCLC has led to an increasing consideration of sublobar resection for patients with small-sized stage I NSCLC [8].

Several authors have identified the following three points concerning the usefulness of sublobar resection: 1) safety of the perioperative course $[9,10]$; 2) effectiveness of preservation of pulmonary function compared with lobectomy $[11,12]$; and 3) oncologic outcome equivalent to lobectomy [13-15]. However, these studies are clearly heterogeneous with regard to the risk status of the patients, the tumour biology and the mode of sublobar resection. In addition, a population-based analysis demonstrated that lobectomy conferred a significant survival advantage compared with segmentectomy [16]. However, a meta-analysis and a review each concluded that segmentectomy provides a survival rate similar to that with lobectomy for tumours $\leqslant 2 \mathrm{~cm}$ in size, but not for larger tumours [17, 18]. Thus, the role of sublobar resection in the treatment of NSCLC has not been fully evaluated [19]. Accordingly, we need to comprehensively understand the present and updated roles of sublobar resection for patients with NSCLC.

\section{Advantages of sublobar resection}

If less lung parenchyma is removed by sublobar resection, patients should benefit from both improved perioperative outcomes (morbidity and mortality) and preserved pulmonary function.

Altorki et al. [9] reviewed the outcomes of 347 patients diagnosed with lung cancer who underwent lobectomy $(n=294)$ or sublobar resection $(n=53)$ for NSCLC in the International Early Lung Cancer Action Program from 1993 to 2011 . The mortality rate was $0 \%$ for the sublobar resection group and $1.0 \%$ for the lobectomy group [9]. SCHUCHERT et al. [10] also reviewed the perioperative outcomes of 182 consecutive segmentectomies and 246 lobectomies for early stage NSCLC. They reported morbidity and mortality rates of $32.4 \%$ and $1.1 \%$, respectively, for anatomic segmentectomy, and $33.7 \%$ and $3.3 \%$, respectively, for lobectomy [10]. One report from a Japanese survey gave a mortality rate of $0.1 \%$ for sublobar resection and $0.3 \%$ for lobectomy [20]. Thus, sublobar resection can be performed with perioperative outcomes comparable to or better than those for lobectomy. However, the initial results of the randomised, prospective American College of Surgeons Oncology Group (ACOSOG) Z0030 trials reported morbidity and mortality rates of $46 \%$ and $3 \%$, respectively, in the setting of anatomic segmentectomy, and $37 \%$ and $1 \%$, respectively, in the setting of lobectomy [21].

KEENAN et al. [11] conducted a retrospective study to evaluate the degree of post-operative loss of pulmonary function among patients who underwent segmental resection or lobectomy. They concluded that segmental resection preserved pulmonary function better than lobectomy [11]. HARADA et al. [12] also evaluated the degree of post-operative functional loss in patients who underwent segmentectomy or lobectomy. They concluded that the extent of removed lung parenchyma directly affected post-operative functional loss, even at 6 months post-surgery, and segmentectomy offered significantly better preservation of function than lobectomy [12]. No previous studies have evaluated the degree of post-operative loss of pulmonary function among patients undergoing wedge resection or lobectomy.

Thus, there is still some controversy regarding both the safety of sublobar resection and the preservation of pulmonary function by sublobar resection. Whether or not sublobar resection actually improves 
perioperative outcomes (morbidity and mortality) and preserves pulmonary function should be prospectively evaluated with an adequate number of patients.

\section{Mode of sublobar resection}

Sublobar resection generally refers to wedge resection and segmental resection (segmentectomy). Wedge resection involves the macroscopic removal of the tumour with surrounding normal lung tissue and does not follow anatomical boundaries. In contrast, segmentectomy involves the macroscopic removal of the tumour with the division of vessels and bronchi that belong to a distinct anatomical segment(s). Thus, draining lymphatics and veins of the tumour can be removed and a wider margin of resection compared with wedge resection can be achieved by segmentectomy. This is the greatest benefit with the use of segmentectomy. Theoretically, segmentectomy results in lower recurrence rates than wedge resection. In fact, several studies have demonstrated that segmentectomy is associated with a decreased risk of local recurrence compared with wedge resection $[22,23]$.

However, segmentectomy has some disadvantages compared with wedge resection. Since selective isolation and division of the targeted bronchovascular pedicle for each segment should be performed, segmentectomy inevitably takes longer and carries the possibility of considerable bleeding compared with wedge resection. In fact, the Japanese survey mentioned previously reported an in-hospital mortality rate of $0.3 \%$ for segmentectomy and $0.2 \%$ for wedge resection [20]. Wedge resection might be a less invasive procedure than segmentectomy. Segmentectomy also may not always be technically feasible and is best suited to the left upper lobe (lingula, apicoposterior and anterior segments) and the apical segment of both lower lobes [24, 25]. Thus, the superiority of segmentectomy over wedge resection might depend on several factors, such as the risk status of the patient or the tumour location.

While segmentectomy has been believed to be superior to wedge resection, the potential benefits and limitations of segmentectomy should be fully considered.

\section{Indications for sublobar resection}

Patients with NSCLC who are candidates for surgery are divided into two groups: standard-risk patients and high-risk patients. High-risk patients are those who have poor pulmonary reserve or other limiting factors and who could withstand thoracotomy but not lobectomy. Patients with prior resection and elderly patients, even if they have normal pulmonary function, might also be considered high risk [26-28]. The American College of Surgeons Oncology Group/National Institutes of Health inoperability criteria for lung surgery have often been used to identify high-risk patients [29]. An algorithm for the assessment of cardiopulmonary reserve before lung resection in lung cancer patients, proposed by the European Respiratory Society/European Society of Thoracic Surgery, has also been widely used [30]. However, these approaches cannot identify every high-risk patient since patients with good cardiopulmonary function are sometimes considered to be poor surgical candidates due to other non-cardiopulmonary comorbidities. As an exceptional case, some patients with a very low pre-operative forced expiratory volume in $1 \mathrm{~s}(\mathrm{FEV} 1)$ or $\mathrm{FEV}$ //forced vital capacity ratio are less likely to lose pulmonary function after lobectomy and may actually see improvements [31]. The chronic obstructive pulmonary disease (COPD) index may be useful for predicting the change in FEV1 after lobectomy. In addition to the former two major criteria, these findings should be considered in patients with severe COPD. Thus, there is still an important issue/controversy in the definition of high-risk patients.

There are two clearly different rationales for the use of sublobar resection in patients with NSCLC; an intentional limited resection and a compromised limited resection. Sublobar resection for standard-risk patients is performed as an intentional limited resection. Intentional limited resection may also include sublobar resection for patients with noninvasive or minimally invasive tumour that is sufficiently curable by limited resection. In contrast, sublobar resection for high-risk patients is performed as a compromised limited resection.

There are crucial differences in the role of sublobar resection as intentional limited resection and as compromised limited resection. In the intentional setting, patients with sublobar resection must have perioperative and oncological outcomes equivalent to those of patients with lobectomy. Patients with intentional sublobar resection must not experience special forms of loco-regional recurrence, which may not occur if they had undergone lobectomy. The importance of adequate hilar/mediastinal staging to avoid erroneous downstaging of the disease cannot be overemphasised. In contrast, oncological outcomes in the compromised setting might be inevitably worse than those in the intentional setting. Therefore, surgeons should focus on the balance between the risks and benefits when deciding upon the mode of resection. They should also consider nonsurgical therapeutic options, such as SBRT or radiofrequency ablation. 


\section{Intentional limited resection}

Only one randomised study, by the Lung Cancer Study Group [32], has compared lobectomy with intentional sublobar resection (segmentectomy or wedge resection) in patients with stage I NSCLC. The results of this study showed a three-fold increase in local recurrence for patients with sublobar resection compared to those with lobectomy, but no significant difference in overall survival [32].

Since then there have been five studies on intentional limited resection performed in Japan (table 1). KODAMA et al. [33] reported the results of intentional limited resection (segmentectomy) for T1 N0 M0 NSCLC at a single institution. The overall 5-year survival rate was $93 \%$ and the local recurrence rate was $8.7 \%$ [33]. TSUBOTA et al. [34] reported the early results of a prospective, multicentre trial of limited surgical resection (segmentectomy) for peripheral NSCLC $\leqslant 2 \mathrm{~cm}$ in size. The overall 5-year survival rate was $91 \%$ and the recurrence rate was $2 \%$ [34]. OKADA et al. [35] also reported the results of a prospective, nonrandomised, multicentre trial that compared sublobar resection $(n=305)$ to lobectomy $(n=262)$ for NSCLC $\leqslant 2 \mathrm{~cm}$ in size. The local recurrence rate was $4.9 \%$ in the sublobar resection group and $6.9 \%$ in the lobectomy group. The disease-free and overall survival rates were similar in the two groups, with 5-year survival rates of $85.9 \%$ and $89.6 \%$, respectively, for the sublobar resection group and $83.4 \%$ and $89.1 \%$, respectively, for the lobectomy group [35]. Furthermore, KoIKE et al. [36] retrospectively reviewed 223 patients with small ( $\leqslant 2 \mathrm{~cm}$ in size) peripheral NSCLC who underwent intentional segmentectomy at their institution. The 5- and 10-year overall survival rates were $89.6 \%$ and $81.0 \%$, respectively. The local recurrence rate was 3.6\% [36]. More recently, TSUTANI et al. [37] reported the results of a multicentre trial that compared intentional segmentectomy $(n=98)$ to lobectomy $(n=383)$ for stage IA adenocarcinoma. The local recurrence rate was $3 \%$ in the segmentectomy group and $4.4 \%$ in the lobectomy group. The recurrence-free and overall survival rates were similar in the two groups, with 3-year survival rates of $91.4 \%$ and $96.9 \%$, respectively, for the segmentectomy group and $87.3 \%$ and $94.1 \%$, respectively, for the lobectomy group [37]. The most crucial point in these studies is intra-operative adequate hilar/mediastinal nodal staging by complete lymph node dissection or nodal sampling. If there were any findings during the operation, such as lymph node metastasis or a surgical margin that was insufficient for sublobar resection, the operative procedure was altered to lobectomy.

There are two ongoing randomised, phase III trials of lobectomy versus sublobar resection for small $(\leqslant 2 \mathrm{~cm})$ peripheral NSCLC; JCOG 0802 and CALGB 140503, respectively. The Japanese phase III study is evaluating the non-inferiority in overall survival of segmentectomy compared with lobectomy in patients with small-sized $(\leqslant 2 \mathrm{~cm}$ ) peripheral NSCLC, excluding radiologically determined noninvasive cancer. The primary end-point is overall survival. The planned sample size is 1100 patients, with 550 cases per arm [38]. In the USA, a recently initiated randomised controlled trial in approximately 1200 patients is comparing lobectomy to limited resection (segmentectomy or wedge resection) for the treatment of patients with NSCLC $\leqslant 2 \mathrm{~cm}$ in size. The primary objective is to determine whether disease-free survival after sublobar resection is non-inferior to that after lobectomy in patients with small peripheral ( $\leqslant 2 \mathrm{~cm})$ NSCLC [39].

Sublobar resection for a tumour without metastatic potential or with low metastatic potential is also considered to be an intentional limited resection. Adenocarcinoma in situ, which was formerly known as bronchioloalveolar carcinoma, is a small $(\leqslant 3 \mathrm{~cm})$ solitary adenocarcinoma that consists of purely lepidic growth without invasive foci. Minimally invasive adenocarcinoma is a small $(\leqslant 3 \mathrm{~cm})$ solitary adenocarcinoma with invasive foci $\leqslant 5 \mathrm{~mm}$ [40]. Adenocarcinoma in situ and minimally invasive adenocarcinoma represent a completely separate group of adenocarcinomas since they show $100 \%$, or near$100 \%$, disease-specific survival, respectively, if completely resected [41, 42]. Therefore, adenocarcinoma in situ and minimally invasive adenocarcinoma are candidates for the use of intentional sublobar resection.

KOIKE et al. [43] conducted a prospective, phase II study to evaluate limited resection for noninvasive bronchioloalveolar carcinoma diagnosed by intra-operative pathological examination. In their study, 44 patients underwent wedge resection and two underwent segmentectomy. Permanent pathological examination revealed that three patients had primary lung adenocarcinomas other than noninvasive bronchioloalveolar carcinoma. The predictive value of intra-operative pathological examination for noninvasive bronchioloalveolar carcinoma diagnosis was $94 \%$. The 5 -year cancer-specific survival rate was $100 \%$ [43]. Other reports have demonstrated a similar outcome $[44,45]$. Ground-glass opacity with little or no consolidation on high-resolution represents adenocarcinoma in situ or minimally invasive adenocarcinoma. SUZUKI et al. [46] reported the results of the JCOG0201 study and demonstrated that radiological noninvasive peripheral lung adenocarcinoma could be defined as an adenocarcinoma $\leqslant 2.0 \mathrm{~cm}$ with $\leqslant 0.25$ consolidation on high-resolution CT. In addition, the phase II trial JCOG0804 was conducted to evaluate limited surgical resection for "radiological early lung cancer" as defined by JCOG0201. Patient accrual is now complete. Over the next 10 years, this study will determine whether their criteria for early 


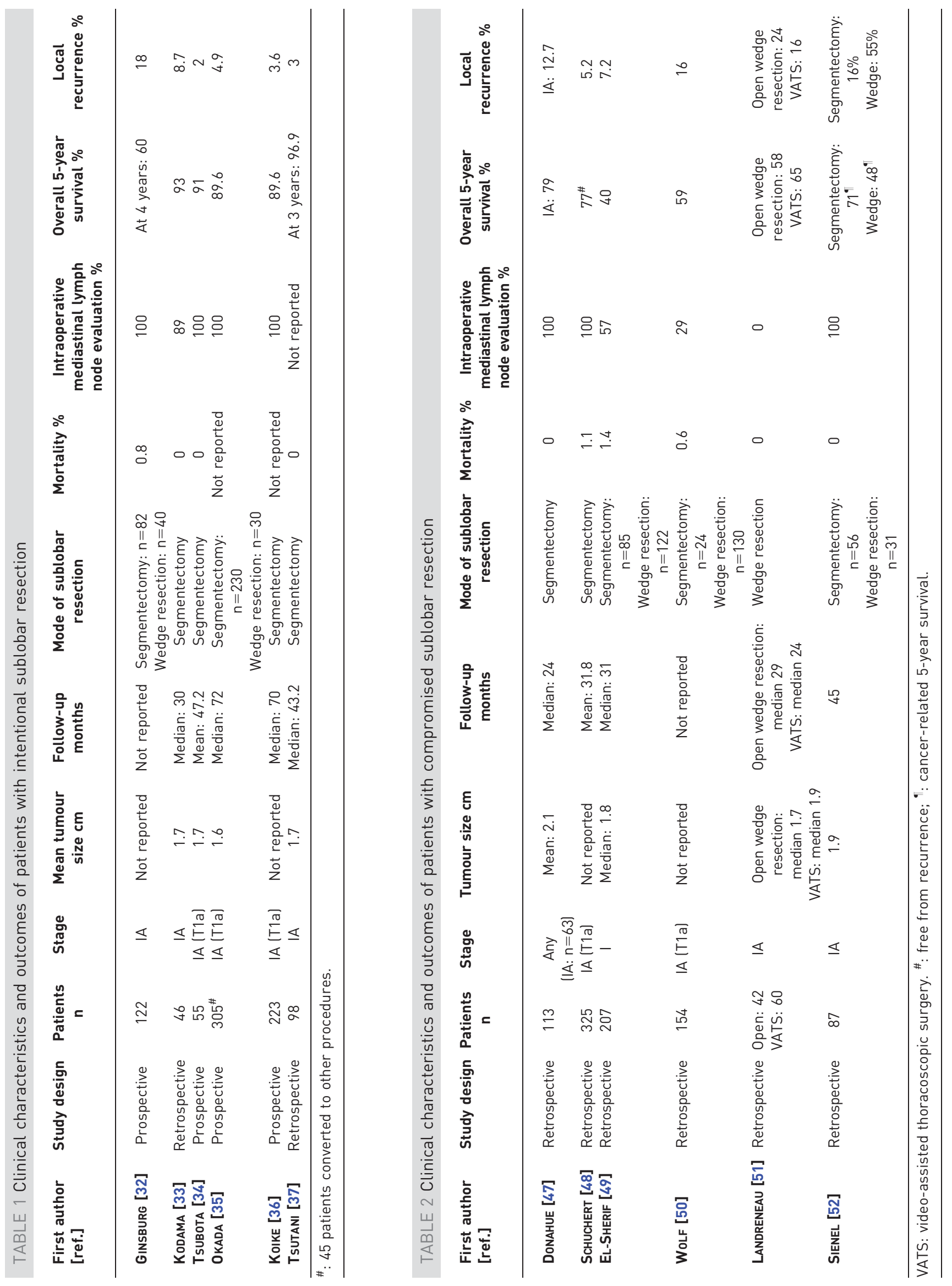


lung adenocarcinomas are adequate. Thus, adenocarcinoma in situ and minimally invasive adenocarcinoma represent ideal lesions for which limited resection gives results equivalent to lobectomy.

At present, intentional limited resection is mainly performed for small-sized NSCLC $\leqslant 2 \mathrm{~cm}$, and the favourable results of intentional sublobar resection have been based on several nonrandomised studies. We need to await the results of ongoing randomised trials that are assessing the utility of sublobar resection for small-sized NSCLC; they will likely provide important contributions to the role of intentional limited resection. Until then, lobectomy remains the standard procedure even for small NSCLC and sublobar resection should still be performed only in a clinical trial setting. In contrast, intentional limited resection is appropriate for small NSCLC that present as ground-glass opacities or semi-solid lesions, which represents adenocarcinoma in situ or minimally invasive adenocarcinoma under the condition of proper radiological and pathological examination.

\section{Compromised limited resection}

High-risk patients who are not eligible for lobectomy because of poor pulmonary reserve or other major comorbidities are still candidates for surgery. Sublobar resection, either segmentectomy or wedge resection, is appropriate for these patients. There have been three types of reports on the results of sublobar resection as a compromised limited resection: those that included only segmentectomy, those that included both segmentectomy and wedge resection, and those that included only wedge resection. The results of the compromised limited resection are summarised in table 2.

DONAHUE et al. [47] reported the results of anatomic segmentectomy in 113 compromised patients with NSCLC. The indications for segmentectomy were poor pulmonary function, comorbidities or the surgeon's judgment. The local recurrence rate was $12.7 \%$ and the 5 -year recurrence-free survival rate was $79 \%$ for stage IA patients with T1a lesion $(n=63)$ [47]. SCHUCHERT et al. [48] also conducted a similar study with 785 patients who underwent anatomic segmentectomy. The decision to perform segmentectomy was based on tumour size, location (peripheral lesions $\leqslant 2 \mathrm{~cm}$ in size), the patient's underlying cardiopulmonary status and other relevant comorbidities. The local recurrence rate was $5.2 \%$ and the 5 -year recurrence-free survival rate was $77 \%$ for 325 patients with stage IA NSCLC [48]. Thus, the 5-year survival rate of patients with compromised segmentectomy seems to be $5-10 \%$ lower than that of patients with intentional segmentectomy. EL-SHERIF et al. [49] retrospectively reviewed the outcomes of 784 patients with stage I NSCLC treated with lobectomy $(n=577)$ or sublobar resection (segmentectomy: $n=85$, wedge resection: $\mathrm{n}=122$ ). Sublobar resection was performed for patients with cardiopulmonary impairment that precluded lobectomy. The local recurrence rate was $7.2 \%$ for the sublobar resection group and $4.2 \%$ for the lobectomy group. The 5-year disease-free survival rate was $40 \%$ for the sublobar resection group and $54 \%$ for the lobectomy group [49]. Wolf et al. [50] also retrospectively reviewed the outcomes of 238 patients with clinical T1a N0 M0 NSCLC treated with lobectomy $(n=84)$ or sublobar resection (segmentectomy: $n=24$; wedge resection: $n=130$ ). Patients who underwent sublobar resection were older and had worse preoperative pulmonary function. The local recurrence rate was $16 \%$ for the sublobar resection group and $8 \%$ for the lobectomy group. The 5-year survival rate was 59\% for the sublobar resection group and $80 \%$ for the lobectomy group [50].

LANDRENEAU et al. [51] analysed the outcomes of a series of patients with peripheral stage IA (T1 N0 M0) NSCLC treated with open lobectomy $(n=117)$, open wedge resection $(n=42)$ or video-assisted thoracoscopic surgery wedge resection $(n=60)$. Patients with a significant impairment of their physiologic condition underwent wedge resection based on the surgeon's decision. There was a trend toward increased local recurrence in the wedge resection group (16\%) compared to the open lobectomy group (9\%), although this difference was not statistically significant. While there was no significant difference in overall survival between patients treated with video-assisted thoracoscopic surgery wedge resection compared to open lobectomy, there was a significant decrease in overall survival for patients with open wedge resection. The 5 -year survival rates were $58 \%$ for patients with open wedge resection, $65 \%$ for those with videoassisted thoracoscopic surgery wedge resection and 70\% for those with lobectomy [51].

Thus, several reports have suggested that high-risk patients with wedge resection have a worse prognosis than those with segmentectomy, although there is considerable patient selection bias with regard to the physiological status of the patient and the tumour biology.

A few reports have compared segmentectomy to wedge resection for high-risk patients with NSCLC. SIENEL et al. [52] reported the results of sublobar resection (segmentectomy: $n=56$; wedge resection: $n=31$ ) in patients with stage IA NSCLC. The local recurrence rate was $16 \%$ for the segmentectomy group and $55 \%$ for the wedge resection group. The cancer-related 5-year survival rate was $71 \%$ for the segmentectomy group and $48 \%$ for the wedge resection group [52]. 
Several authors have reported the usefulness of adjuvant radiotherapy or brachytherapy for reducing local recurrence after sublobar resection. FERNANDO et al. [53] concluded that intraoperative brachytherapy might reduce local recurrence. More recently, the American College of Surgeons Oncology Group conducted a randomised study (Z4032) that compared sublobar resection alone to sublobar resection with brachytherapy for high-risk operable patients with NSCLC [54].

Patients with compromised limited resection tend to have a higher recurrence rate and a lower survival rate than those with intentional limited resection. The superiority of segmentectomy to wedge resection in these populations has not yet been clarified because there has been no formal comparison of segmentectomy to wedge resection. Furthermore, there has been no comparison of sublobar resection with nonsurgical treatment, such as SBRT or radiofrequency ablation. Prospective, randomised studies will be needed to fully delineate the utility of sublobar resection, SBRT or radiofrequency ablation in high-risk patients with NSCLC.

\section{Stereotactic radiotherapy or stereotactic ablative radiotherapy}

The gold standard for treating stage I NSCLC is a lobectomy with systematic nodal dissection [49]. However, due to comorbidities this treatment is deemed unfeasible in a significant proportion of patients, for which alternatives were sought [50-53]. Pioneered in Japan, SBRT or SABR has been introduced in clinical practice in the past decade [50-53].

In SABR, a few fractions of very high doses of radiation are delivered to small tumours that are located in lung regions able to sustain an ablative dose of radiotherapy. In most studies, so-called "central" tumours, e.g. those remote from the more susceptible central mediastinal structures such as the main bronchi, large vessels and the oesophagus, are not included [54]. Although some centres advocate SBRT in central tumours [55], this is the subject of an ongoing phase II trial, LUNG-TECH, led by the European Organisation for Research and Treatment of Cancer. The advantages of SABR compared with conventional irradiation are a short overall treatment time (1-2 weeks) and a very high biologically effective dose. SABR offers the possibility to minimise the time investment for treatment and toxicity, which is of obvious benefit for medically inoperable patients suffering from major comorbidities or advanced age. SABR requires highly specialised radiotherapy preparation and execution techniques including four-dimensional respiratory correlated CT scans and image-guided radiotherapy.

45 studies concerning SABR in early stage NSCLC have been reported [56]. 13 studies including 996 patients were prospective, and 32 studies including 2645 patients were retrospective.

The results of the prospective trials with SABR are summarised in table 3 [52, 57-73]. The 2-year overall survival was $65.2 \%$, with a 2 -year local tumour control rate of $90.4 \%$. When all 45 studies on SABR, totalling 3641 patients, were considered, the 2-year overall survival was 70\% (95\% CI 67-92\%) and the average local tumour control rate after 2 years was 91\% (95\% CI 90-93\%) [56]. Even though the patient selection may have been different between studies and older reports used inevitably simpler techniques, neither the 2-year overall survival nor the 2-year local tumour control rate different significantly [56]. The 2-year local tumour control rate was 91\% (95\% CI 89-93\%) for linear accelerator-based techniques, $88 \%$ (95\% CI 78-94\%) for Cyberknife (Accuracy Inc., Sunnyvale, CA, USA), and 80\% (95\% CI 68-91\%) for other technologies. The 2-year overall survival was 69\% (95\% CI 66-71\%) for linear accelerator-based techniques, 73\% (95\% CI 61-83\%) for Cyberknife and 75\% (95\% CI 65-83\%) for other technologies. These figures remained stable for at least 5 years [68]. After 5 years, the loco-regional recurrences rates were $\sim 15 \%$, with $\sim 20 \%$ of the patients developing distant metastases [68]. Considering the robustness of these findings within many studies worldwide, it is fair to state that SBRT has been proven to be comparable to outcome of lobectomy and lymph node dissection $[69,70]$. Indeed, in the International Association for the Study of Lung Cancer database, which also comprises a large, relatively non-selected group of patients, the 2 -year overall survival was 68\% (66-70\%) [56].

The toxicity of SABR is favourable; $<10 \%$ of the patients experienced important side-effects, which also makes SABR suitable for elderly and/or frail patients $[68,71]$. Pulmonary function (FEV1 and diffusing capacity of the lung for carbon monoxide (DLCO)) does not deteriorate after SABR, even in individuals with a poor baseline lung function [72]. Therefore, SABR is not contraindicated in patients with severe COPD [72].

Therefore, SABR has become the treatment of choice for inoperable patients with stage I NSCLC and is possibly competitive with lobectomy.

In some patients, mainly due to poor pulmonary function or other comorbidities, a pathological diagnosis of the tumour cannot be obtained. In these cases, algorithms have been developed that allow the a priori criteria for malignancy to be calculated. In Europe, the probability for malignancy is $>95 \%$ when a lesion is new, has CT characteristics of malignancy and is 2-fluoro-2-deoxy-D-glucose (FDG) avid [74]. In the 


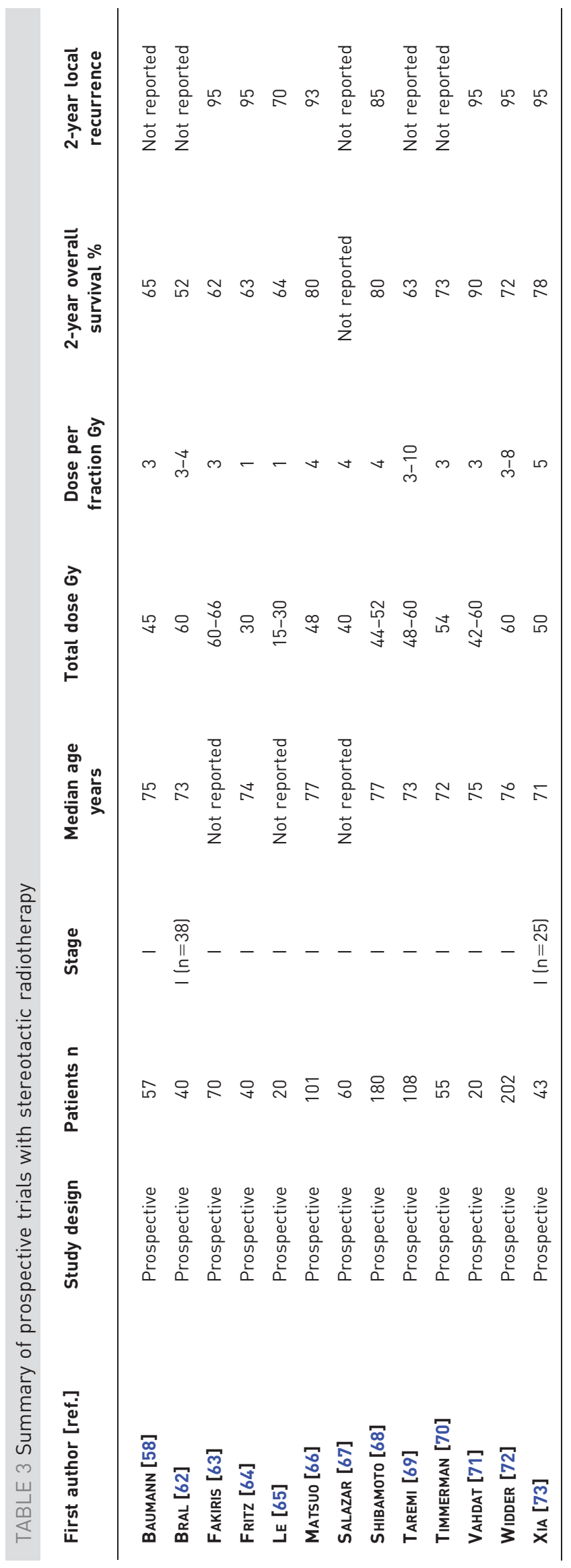


absence of a clear alternative diagnosis, SABR may be delivered without pathology [74]. Nevertheless, it should be stressed that a pathological diagnosis should always be preferentially obtained whenever possible.

The prognosis of patients treated with SABR with or without a pathological diagnosis is similar [75]. This supports the use of the above-mentioned algorithm, at least in Europe where other diseases mimicking malignant coin lesions are very rare.

Historically, patients referred for SABR were the older and/or frailer patient group. With the recognised success of SABR, more borderline operable or even operable patients that refuse surgery are being treated with radiotherapy. In both cases, thorough staging is advised. Indeed, besides the detection of distant metastases by a whole-body FDG-positron emission tomography-CT scan, mediastinal and hilar lymph node involvement should be identified as it will alter the local and systemic treatment. As $\sim 15 \%$ of the patients fail in the lymph nodes [76], endobronchial ultrasound/endoscopic ultrasound and, when appropriate, mediastinoscopy should be considered, taking into account the general condition and the comorbidities of the patient.

In particular, in patients who are potential candidates for salvage surgery early detection of local recurrences after SABR are particularly challenging. All patients show persistent and even fluctuating changes in the lungs [77]. Therefore, it is important to discuss follow-up scans multidisciplinary with experts that take into account not only tumour size, but also other morphological criteria [77]. FDG-positron emission tomography-CT scans may be falsely positive after SABR and should be interpreted in the context of other diagnostic criteria [77].

\section{Sublobar resection, SABR or radiofrequency ablation?}

In recent years, sublobar resections have gained popularity as a way to spare lung tissue. Randomised trials comparing lobectomy to sublobar resections are underway. At the time of writing, no phase III comparisons have been reported on SABR versus sublobar resections.

In an attempt to indirectly compare SABR to sublobar resections and radiofrequency ablation, CRABTREE et al. [78] compared the selection criteria and short-term outcomes of three prospective clinical trials using: SABR in the Radiation Therapy Oncology Group (RTOG) trial 0236), sublobar resection in the ACOSOG trial Z4032, and radiofrequency ablation in the ACOSOG trial Z4033. Although the patients in ACOSOG Z4033 were older ( $75.6 \pm 7.5$ years) than those in RTOG 0236 ( $72.5 \pm 8.8$ years) and ACOSOG Z4032 $(70.2 \pm 8.5$ years; $\mathrm{p}=0.0003)$, the pre-treatment $\mathrm{FEV} 1$ was $61.3 \pm 33.4 \%, 53.8 \pm 19.6 \%$ and $48.8 \pm 20.3 \%$ for RTOG 0236, ACOSOG Z4032 and ACOSOG Z4033 ( $\mathrm{p}=0.15)$, respectively. The pre-treatment DLCO was $61.6 \pm 30.2 \%, 46.4 \pm 15.6 \%$ and $43.7 \pm 18.0 \%$ for RTOG 0236, ACOSOG Z4032 and ACOSOG Z4033 $(\mathrm{p}=0.001)$, respectively. The overall 90 -day mortality for SABR, sublobar resection and radiofrequency ablation was $0 \%, 2.4 \%$ (five out of 211 ) and $2.0 \%$ (one out of 51 ) $(\mathrm{p}=0.5$ ), respectively. Overall, the unadjusted 30-day grade 3 or more adverse events were more common with sublobar resection than with SABR $(28 \%$ versus $9.1 \%, \mathrm{p}=0.004)$, although there was no difference between the two groups at 90 days. Of the patients with clinical stage IA in ACOSOG Z4032, 29.3\% had a more advanced pathological stage at surgery. A propensity-matched comparison showed no difference between SABR and sublobar resection for 30-day grade 3 or more adverse events (OR 2.37 (95\% CI 0.75-9.90); p=0.18). Neither FEV1 nor DLCO declined after SABR or sublobar resection. There were not enough data on radiofrequency ablation outcomes to draw conclusions.

\section{Conclusions}

Lobectomy and systematic nodal dissection are still the standard for small-size $(<3 \mathrm{~cm})$ NSCLC. Nonrandomised trials suggest that a segmentectomy may result in local control rates that are similar to lobectomy. Nonsurgical approaches, such as SABR, consistently result in local control rates of $\sim 90 \%$ and survival rates that are comparable to lobectomy. Short-term results for radiofrequency ablation are also comparable to sublobar resection and SABR, but there is a lack of long-term results. Until the results of randomised clinical trials become available, both SABR and sublobar resection may be offered to appropriate patients.

\section{References}

1 Howington JA, Blum MG, Chang AC, et al. Treatment of stage I and II non-small cell lung cancer: diagnosis and management of lung cancer, 3rd ed: American College of Chest Physicians evidence-based clinical practice guidelines. Chest 2013; 143: Suppl. 5, e278S-313S.

2 National Comprehensive Cancer Network. Non-small cell lung cancer NCCN Guidelines. Version 2, 2014. www.nccn.org/professionals/physician_gls/pdf/nhl.pdf

3 Churchill ED, Sweet RH, Scannell JG, et al. Further studies in the surgical management of carcinoma of the lung. J Thorac Surg 1958; 36: 301-308. 
Bonfils-Roberts EA, Claggett OT. Contemporary indication for pulmonary segmental resections. J Thorac Surg 1972; 63: 433-438.

5 Jensik RJ, Faber LP, Milloy FJ, et al. Segmental resection for lung cancer. A fifteen year experience. $J$ Thorac Cardiovasc Surg 1973; 66: 563-572.

6 Read RC, Yoder G, Schaeffer RC, et al. Survival after conservative resection for TIN0M0 non-small cell lung cancer. Ann Thorac Surg 1990; 49: 242-247.

7 Henschke CI, Yankelevitz DF, Altorki NK. The role of CT screening for lung cancer. Thorac Surg Clin 2007; 17: $137-142$.

8 Rami-Porta R, Ball D, Crowley JJ, et al. The IASLC Lung Cancer Staging Project: proposals for revision of the T descriptors in the forthcoming (seventh) edition of the TNM classification of lung cancer. J Thorac Oncol 2007; 2: 593-602.

9 Altorki NK, Yip R, Hanaoka T, et al. Sublobar resection is equivalent to lobectomy for clinical stage 1A lung cancer in solid nodules. J Thorac Cardiovasc Surg 2014; 147: 754-762.

10 Schuchert MJ, Pettiford BL, Keeley S, et al. Anatomic segmentectomy in the treatment of stage I non-small cell lung cancer. Ann Thorac Surg 2007; 84: 926-933.

11 Keenan RJ, Landreneau RJ, Maley RH Jr, et al. Segmental resection spares pulmonary function in patients with stage I lung cancer. Ann Thorac Surg 2004; 78: 228-233.

12 Harada H, Okada M, Sakamoto T, et al. Functional advantage after radical segmentectomy versus lobectomy for lung cancer. Ann Thorac Surg 2005; 80: 2041-2045.

13 Pettiford BL, Schuchert MJ, Santos R, et al. Role of sublobar resection (segmentectomy and wedge resection) in the surgical management of non-small cell lung cancer. Thorac Surg Clin 2007; 17: 175-190.

14 Okumura M, Goto M, Ideguchi K, et al. Factors associated with outcome of segmentectomy for non-small cell lung cancer: long-term follow-up study at a single institution in Japan. Lung Cancer 2007; 58: $231-237$.

15 Wisnivesky JP, Henschke CI, Swanson S, et al. Limited resection for the treatment of patients with stage IA lung cancer. Ann Surg 2010; 251: 550-554.

16 Whitson BA, Groth SS, Andrade RS, et al. Survival after lobectomy versus segmentectomy for stage I non-small cell lung cancer: a population-based analysis. Ann Thorac Surg 2011; 92: 1943-1950.

17 Fan J, Wang L, Jiang GN, et al. Sublobectomy versus lobectomy for stage I non-small-cell lung cancer, a metaanalysis of published studies. Ann Surg Oncol 2012; 19: 661-668.

18 Rami-Porta R, Tsuboi M. Sublobar resection for lung cancer. Eur Respir J 2009; 33: 426-435.

19 Blasberg JD, Pass HI, Donington JS. Sublobar resection: a movement from the Lung Cancer Study Group. J Thorac Oncol 2010; 5: 1583-1593.

20 Amano J, Kuwano H, Yokomise H. Thoracic and cardiovascular surgery in Japan during 2011: Annual report by The Japanese Association for Thoracic Surgery. Gen Thorac Cardiovasc Surg 2013; 61: 578-607.

21 Allen MS, Darling GE, Pechet TTV, et al. Morbidity and mortality of major pulmonary resections in patients with early-stage lung cancer: initial results of the randomized, prospective ACOSOG Z0030 trial. Ann Thorac Surg 2006; 81: 1013-1020.

22 El-Sherif A, Fernando HC, Santos R, et al. Margin and local recurrence after sublobar resection of non-small cell lung cancer. Ann Surg Oncol 2007; 14: 2400-2405.

23 Koike T, Koike T, Yoshiya K, et al. Risk factor analysis of locoregional recurrence after sublobar resection in patients with clinical stage IA non-small cell lung cancer. J Thorac Cardiovasc Surg 2013; 146: 372-378.

24 Sienel W, Stremmel C, Kirschbaum A, et al. Frequency of local recurrence following segmentectomy of stage IA non-small cell lung cancer is influenced by segment localisation and width of resection margins-implications for patient selection for segmentectomy. Eur J Cardiothorac Surg 2007; 31: 522-527.

25 Lim E, Baldwin D, Beckles M, et al. Guidelines on the radical management of patients with lung cancer. Thorax 2010; 65: Suppl. 3, iiil-iii27.

26 Okami J, Ito Y, Higashiyama M, et al. Sublobar resection provides an equivalent survival after lobectomy in elderly patients with early lung cancer. Ann Thorac Surg 2010; 90: 1651-1656.

27 Hamaji M, Allen MS, Cassivi SD, et al. Surgical treatment of metachronous second primary lung cancer after complete resection of non-small cell lung cancer. J Thorac Cardiovasc Surg 2013; 145: 683-690.

28 Zuin A, Andriolo LG, Marulli G. Is lobectomy really more effective than sublobar resection in the surgical treatment of second primary lung cancer? Eur J Cardiothorac Surg 2013; 44: e120-e125.

29 Zemlyak A, Moore WH, Bilfinger TV. Comparison of survival after sublobar resections and ablative therapies for stage I non-small cell lung cancer. J Am Coll Surg 2010; 211: 68-72.

30 Brunelli A, Charloux A, Bolliger CT, et al. ERS/ESTS clinical guidelines on fitness for radical therapy in lung cancer patients (surgery and chemo-radiotherapy). Eur Respir J 2009; 34: 17-41.

31 Korst RJ, Ginsberg RJ, Ailawadi M, et al. Lobectomy improves ventilatory function in selected patients with severe COPD. Ann Thorac Surg 1998; 66: 898-902.

32 Ginsberg RJ, Rubenstein LV. Randomized trial of lobectomy versus limited resection for T1N0 non-small cell lung cancer. Lung Cancer Study Group. Ann Thorac Surg 1995; 60: 615-622.

33 Kodama K, Doi O, Higashiyama M, et al. Intentional limited resection for selected patients with T1 N0 M0 nonsmall-cell lung cancer: a single-institution study. J Thorac Cardiovasc Surg 1997; 114: 347-353.

34 Tsubota N, Ayabe K, Doi O, et al. Ongoing prospective study of segmentectomy for small lung tumors. Study Group of Extended Segmentectomy for Small Lung Tumor. Ann Thorac Surg 1998; 66: 1787-1790.

35 Okada M, Koike T, Higashiyama M, et al. Radical sublobar resection for small-sized non-small cell lung cancer: a multicenter study. J Thorac Cardiovasc Surg 2006; 132: 769-775.

36 Koike T, Koike T, Yamato Y, et al. Prognostic predictors in non-small cell lung cancer patients undergoing intentional segmentectomy. Ann Thorac Surg 2012; 93: 1788-1794.

37 Tsutani Y, Miyata Y, Nakayama H, et al. Oncologic outcomes of segmentectomy compared with lobectomy for clinical stage IA lung adenocarcinoma: propensity score-matched analysis in a multicenter study. J Thorac Cardiovasc Surg 2013; 146: 358-364.

38 Nakamura K, Saji H, Nakajima R, et al. A. phase III randomized trial of lobectomy versus limited resection for small-sized peripheral non-small cell lung cancer (JCOG0802/WJOG4607L). Jpn J Clin Oncol 2010; 40: 271-274. 
39 National Cancer Institute at the National Institutes of Health. Comparison of different types of surgery in treating patients with stage IA non-small cell lung cancer. www.cancer.gov/clinicaltrials/search/view?cdrid $=555324 \&$ version $=$ HealthProfessional Date last updated: October 17, 2013. Date last accessed: January 30, 2014.

40 Travis WD, Brambilla E, Noguchi M, et al. International Association for the Study of Lung Cancer/American Thoracic Society/European Respiratory Society international multidisciplinary classification of lung adenocarcinoma. J Thorac Oncol 2011; 6: 244-285.

41 Travis WD, Brambilla E, Van Schil P, et al. Paradigm shifts in lung cancer as defined in the new IASLC/ATS/ERS lung adenocarcinoma classification. Eur Respir J 2011; 38: 239-243.

42 Van Schil PE, Asamura H, Rusch VW. Surgical implications of the new IASLC/ATS/ERS adenocarcinoma classification. Eur Respir J 2012; 39: 478-486.

43 Koike T, Togashi K, Shirato T, et al. Limited resection for noninvasive bronchioloalveolar carcinoma diagnosed by intraoperative pathologic examination. Ann Thorac Surg 2009; 88: 1106-1111.

44 Yamato $\mathrm{Y}$, Tsuchida $\mathrm{M}$, Watanabe $\mathrm{T}$, et al. Early results of a prospective study of limited resection for bronchioloalveolar adenocarcinoma of the lung. Ann Thorac Surg 2001; 71: 971-974.

45 Watanabe S, Watanabe T, Arai K, et al. Results of wedge resection for focal bronchioloalveolar carcinoma showing pure ground-glass attenuation on computed tomography. Ann Thorac Surg 2002; 73: 1071-1075.

46 Suzuki K, Koike T, Asakawa T, et al. A prospective radiological study of thin-section computed tomography to predict pathological noninvasiveness in peripheral clinical IA lung cancer (Japan Clinical Oncology Group 0201). J Thorac Oncol 2011; 6: 751-756.

47 Donahue JM, Morse CR, Wigle DA, et al. Oncologic efficacy of anatomic segmentectomy in stage IA lung cancer patients with T1a tumors. Ann Thorac Surg 2012; 93: 381-387.

48 Schuchert MJ, Abbas G, Awais O, et al. Anatomic segmentectomy for the solitary pulmonary nodule and early-stage lung cancer. Ann Thorac Surg 2012; 93: 1780-1785.

49 El-Sherif A, Gooding WE, Santos R, et al. Outcomes of sublobar resection versus lobectomy for stage I non-small cell lung cancer: a 13-year analysis. Ann Thorac Surg 2006; 82: 408-415.

50 Wolf AS, Richards WG, Jaklitsch MT, et al. Lobectomy versus sublobar resection for small (2 cm or less) non-small cell lung cancers. Ann Thorac Surg 2011; 92: 1819-1823.

51 Landreneau RJ, Sugarbaker DJ, Mack MJ, et al. Wedge resection versus lobectomy for stage I (T1 N0 M0) nonsmall-cell lung cancer. J Thorac Cardiovasc Surg 1997; 113: 691-698.

52 Sienel W, Dango S, Kirschbaum A, et al. Sublobar resections in stage IA non-small cell lung cancer: segmentectomies result in significantly better cancer-related survival than wedge resections. Eur J Cardiothorac Surg 2008; 33: 728-734.

53 Fernando HC, Santos RS, Benfield JR, et al. Lobar and sublobar resection with and without brachytherapy for small stage IA nonsmall cell lung cancer. J Thorac Cardiovasc Surg 2005; 129: 261-267.

54 Kent M, Landreneau R, Mandrekar S, et al. Segmentectomy versus wedge resection for non-small cell lung cancer in high-risk operable patients. Ann Thorac Surg 2013; 96: 1747-1755.

55 Vansteenkiste J, De Ruysscher D, Eberhardt WE, et al. Early and locally advanced non-small-cell lung cancer (NSCLC): ESMO Clinical Practice Guidelines for diagnosis, treatment and follow-up. Ann Oncol 2013; 24: Suppl. 6, vi89-vi98.

56 Onishi H, Araki T. Stereotactic body radiation therapy for stage I non-small-cell lung cancer: a historical overview of clinical studies. Jpn J Clin Oncol 2013; 43: 345-350.

57 Iyengar P, Timmerman RD. Stereotactic ablative radiotherapy for non-small cell lung cancer: rationale and outcomes. J Natl Compr Canc Netw 2012; 10: 1514-1520.

58 Baumann P, Nyman J, Hoyer M, et al. Outcome in a prospective phase II trial of medically inoperable stage I nonsmall-cell lung cancer patients treated with stereotactic body radiotherapy. J Clin Oncol 2009; 27: 3290-3296.

59 Senthi S, Haasbeek CJ, Slotman BJ, et al. Outcomes of stereotactic ablative radiotherapy for central lung tumours: a systematic review. Radiother Oncol 2013; 106: 276-282.

60 Nestle U, Faivre-Finn C, De Ruysscher D, et al. Stereotactic body radiotherapy (SBRT) in central non-small cell lung cancer (NSCLC): solid evidence or "no-go"? Radiother Oncol 2013; 109: 178-179.

61 Soldà F, Lodge M, Ashley S, et al. Stereotactic radiotherapy (SABR) for the treatment of primary non-small cell lung cancer: systematic review and comparison with a surgical cohort. Radiother Oncol 2013; 109: 1-7.

62 Bral S, Gevaert T, Linthout N, et al. Prospective, risk-adapted strategy of stereotactic body radiotherapy for earlystage non-small-cell lung cancer: results of a Phase II trial. Int J Radiat Oncol Biol Phys 2011; 80: 1343-1349.

63 Fakiris AJ, McGarry RC, Yiannoutsos CT, et al. Stereotactic body radiation therapy for early-stage non-small-cell lung carcinoma: four-year results of a prospective phase II study. Int J Radiat Oncol Biol Phys 2009; 75: 677-682.

64 Fritz P, Kraus HJ, Blaschke T, et al. Stereotactic, high single-dose irradiation of stage I non-small cell lung cancer (NSCLC) using four-dimensional CT scans for treatment planning. Lung Cancer 2008; 60: 193-199.

65 Le QT, Loo BW, Ho A, et al. Results of a phase I dose-escalation study using single-fraction stereotactic radiotherapy for lung tumors. $J$ Thorac Oncol 2006; 1: 802-809.

66 Matsuo Y, Shibuya K, Nagata Y, et al. Prognostic factors in stereotactic body radiotherapy for non-small-cell lung cancer. Int J Radiat Oncol Biol Phys 2011; 79: 1104-1111.

67 Salazar OM, Sandhu TS, Lattin PB, et al. Once-weekly, high-dose stereotactic body radiotherapy for lung cancer: 6year analysis of 60 early-stage, 42 locally advanced, and 7 metastatic lung cancers. Int J Radiat Oncol Biol Phys 2008; 72: 707-715.

68 Shibamoto Y, Hashizume C, Baba F, et al. Stereotactic body radiotherapy using a radiobiology-based regimen for stage I nonsmall cell lung cancer: a multicenter study. Cancer 2012; 118: 2078-2084.

69 Taremi M, Hope A, Dahele M, et al. Stereotactic body radiotherapy for medically inoperable lung cancer: prospective, single-center study of 108 consecutive patients. Int J Radiat Oncol Biol Phys 2012; 82: 967-973.

70 Timmerman R, Paulus R, Galvin J, et al. Stereotactic body radiation therapy for inoperable early stage lung cancer. JAMA 2010; 303: 1070-1076.

71 Vahdat S, Oermann EK, Collins SP, et al. CyberKnife radiosurgery for inoperable stage IA non-small cell lung cancer: 18F-fluorodeoxyglucose positron emission tomography/computed tomography serial tumor response assessment. J Hematol Oncol 2010; 3: 6. 
72 Widder J, Postmus D, Ubbels JF, et al. Survival and quality of life after stereotactic or 3D-conformal radiotherapy for inoperable early-stage lung cancer. Int J Radiat Oncol Biol Phys 2011; 81: e291-e297.

73 Xia T, Li H, Sun Q, et al. Promising clinical outcome of stereotactic body radiation therapy for patients with inoperable stage I/II non-small-cell lung cancer. Int J Radiat Oncol Biol Phys 2006; 66: 117-125.

74 De Ruysscher D, Faivre-Finn C, Nestle U, et al. European Organisation for Research and Treatment of Cancer recommendations for planning and delivery of high-dose, high-precision radiotherapy for lung cancer. J Clin Oncol 2010; 28: 5301-5310.

75 Verstegen NE, Lagerwaard FJ, Haasbeek CJ, et al. Outcomes of stereotactic ablative radiotherapy following a clinical diagnosis of stage I NSCLC: comparison with a contemporaneous cohort with pathologically proven disease. Radiother Oncol 2011; 101: 250-254.

76 Senthi S, Lagerwaard FJ, Haasbeek CJ, et al. Patterns of disease recurrence after stereotactic ablative radiotherapy for early stage non-small-cell lung cancer: a retrospective analysis. Lancet Oncol 2012; 13: 802-809.

77 Mattonen SA, Huang K, Ward AD, et al. New techniques for assessing response after hypofractionated radiotherapy for lung cancer. J Thorac Dis 2014; 6: 375-386.

78 Crabtree T, Puri V, Timmerman R, et al. Treatment of stage I lung cancer in high-risk and inoperable patients: comparison of prospective clinical trials using stereotactic body radiotherapy (RTOG 0236), sublobar resection (ACOSOG Z4032), and radiofrequency ablation (ACOSOG Z4033). J Thorac Cardiovasc Surg 2013; 145: 692-699. 